\title{
Dark matter halos around isolated ellipticals
}

\author{
E. Memola ${ }^{1,2}$, P. Salucci ${ }^{3}$, and A. Babić ${ }^{3}$ \\ 1 INFN-Istituto Nazionale di Fisica Nucleare, Sezione di Milano Bicocca, Piazza della Scienza 3, 20126 Milano, Italy
2 INAF-Istituto Nazionale di Astrofisica, Osservatorio Astronomico di Brera, via Brera 28, 20121 Milano, Italy
e-mail: elisabetta.memola@mib.infn. it
3 SISSA/ISAS, via Bonomea 265, 34136 Trieste, Italy
}

Received 31 August 2010 / Accepted 20 June 2011

\begin{abstract}
Aims. We investigate the distribution of the luminous and the dark matter components in the isolated ellipticals NGC 7052 and NGC 7785, which are embedded in an emitting hot gas halo, by means of relevant X-ray and photometric data.

Methods. To calculate the dark matter distribution in these rare objects, we performed an improved X-ray analysis of the XMM-Newton data of NGC 7785, and we used former results based on Chandra data of NGC 7052. For each object we also derived the stellar spheroid length scale from the surface photometry and the spheroid stellar mass from an analysis of the galaxy spectral energy distribution.

Results. We find that a dark matter component is present in these objects. It is subdominant and mixed with the luminous matter inside the optical region half-light radius wide, while it dominates the gravitational potential at outer radii. On the whole, the dark halo structure is very similar to that found around spirals of comparable luminosity and it is well reproduced by a Burkert halo, while a Sérsic spheroid accounts well for the baryonic component.
\end{abstract}

Key words. galaxies: elliptical and lenticular, cD - X-rays: galaxies - galaxies: photometry - dark matter

\section{Introduction}

The dynamics of galaxies is influenced, and often dominated, by non-radiating matter, which reveals itself only through a gravitational interaction with the luminous matter. From the study of a large number of disk systems, a one-to-one relation between the (luminous) central object and the massive dark halo that envelopes it has emerged (see Shankar et al. 2006; Mandelbaum et al. 2009). The paradigm is that every galaxy lies within a selfgravitating dark halo of size $R_{\text {vir }}$ and mass $M_{\text {vir }}$ : more specifically, disks of sizes $R_{\mathrm{opt}}$ and luminous masses $M_{\star}$ are embedded in dark halos of virial radii $R_{\mathrm{vir}} \sim 15 R_{\mathrm{opt}}$ and masses $M_{\text {vir }}=3 \times 10^{12}\left(\frac{M_{*}}{2 \times 10^{11} M_{\odot}}\right)^{0.4} M_{\odot}$, where $M_{\odot}$ is the solar mass (Persic et al. 1996; Kormendy \& Freeman 2004; Salucci et al. 2007; Oh et al. 2008; Donato et al. 2009).

Do ellipticals follow a similar scenario (see Bertola et al. 1993; Salucci \& Persic 1997; Tortora et al. 2009)? The answer would be of crucial importance for understanding how galaxies form and perhaps even for the nature of the dark matter (DM) itself. Unfortunately, evidence on the properties of the mass distribution in these objects is presently not conclusive.

Indeed, to derive the mass distribution from stellar kinematics, as is done in spirals where rotation balances gravity, is very challenging. Ellipticals are pressure-supported stellar systems whose orbital structure may also involve some angular momentum content, a degree of triaxiality, and velocity dispersion anisotropies. As is well known, the derivation of the mass distribution from the stellar motions in these systems through Jeans equation and its higher moments is quite complex. It is difficult to obtain the galaxy gravitational potential unambiguously and, following this, the mass distribution. Furthermore, in these systems the luminous matter is very concentrated toward the center, where it dominates the gravitational potential. Therefore, the motions of the main baryonic component in ellipticals are generally a limited tracer of the distribution of the dark matter, which mostly lies outside the stellar spheroid (e.g., Kronawitter et al. 2000). Only recent dynamical measurements reached the DM dominated regions (e.g., Thomas et al. 2009, and references therein; Pu et al. 2010).

Alternative mass tracers (planetary nebulae, ionized globular clusters, and neutral disks) from baryonic components of negligible mass are often a better probe of the total gravitational potential (see Romanowsky 2010, and references therein). In particular, the X-ray emitting hot gas is known to extend out to very large radii and to trace the gravitational potential. This method yields accurate mass profiles (e.g., Forman et al. 1985; O'Sullivan et al. 2007; Zhang et al. 2007) and it is very robust because it provides reliable mass profiles also for highly disturbed objects where the hydrostatic equilibrium is far from being established (Johnson et al. 2009).

This method works best for isolated systems and has the rare advantage that it can probe the gravitational potential from almost the galaxy center out to the halo virial radius. Moreover, to obtain mass profiles of objects that never resided in a cluster or group resolves the question of whether ellipticals possess their own DM halos, or "build them" from the cluster DM, and it allows us to investigate the environmental dependence of the DM distribution in galaxies.

\section{X-ray emitting halo ellipticals}

A regular X-ray morphology is commonly assumed to indicate approximate hydrostatic equilibrium, and the absence of nearby companions leaves the X-ray halo unperturbed. The gravitating mass inside a radius $r, M(r)$, can be derived from the X-ray flux under the assumption that the emitting gas is in hydrostatic 
equilibrium. As is well known, from the gas density and temperature profiles (e.g., Fabricant \& Gorestein 1983) we obtain:

$M(<r)=\frac{k T_{\mathrm{g}}(r) r}{G \mu m_{\mathrm{p}}}\left(\frac{\mathrm{d} \log \rho_{\mathrm{g}}}{\operatorname{dlog} r}+\frac{\mathrm{d} \log T_{\mathrm{g}}(r)}{\mathrm{d} \log r}\right)$,

where $T_{\mathrm{g}}$ is the gas temperature at radius $r, \rho_{\mathrm{g}}$ is the gas density, $k$ is the Boltzmann constant, $G$ is the gravitational constant, $\mu$ is the mean molecular weight that we set at the value of $\mu=0.62$ (Ettori \& Fabian 2006), and $m_{\mathrm{p}}$ is the mass of the proton.

The observed X-ray surface brightness, $\Sigma_{\mathrm{g}}$ is usually fitted by a $\beta$-model (Cavaliere \& Fusco-Femiano 1976):

$\Sigma_{\mathrm{g}} \propto\left[1+\left(r / r_{\mathrm{c}}\right)^{2}\right]^{-\beta / 2}$

that yields

$\rho_{\mathrm{g}} \propto\left[1+\left(r / r_{\mathrm{c}}\right)^{2}\right]^{-3 \beta / 2}$,

with the core radius $r_{\mathrm{c}}$, and the slope $\beta$ as free parameters. For an isothermal gas distribution: $\operatorname{d} \log T_{\mathrm{g}}(r) / \operatorname{dlog} r=0$, therefore, we have, in a spherical case, from the above equations:

$M(r) \propto \frac{\left(r / r_{\mathrm{c}}\right)^{3}}{\left[1+\left(r / r_{\mathrm{c}}\right)^{2}\right]}$.

An isothermal distribution is often found in ellipticals; however, there are cases in which the temperature has a profile that must be considered in Eq. (1), although its contribution is almost always much smaller than that from the gas density gradient (e.g. Fukazawa et al. 2006). In the present work, we have a very low spatial resolution temperature measurement, therefore we are forced to assume that the temperature in the region under analysis, varies linearly with radius. An assumption that, however, agrees well with most of the high-resolution temperature profiles. Then, Eq. (4) or, when the temperature gradient is different from zero, Eq. (1) yields the gravitating mass as a function of the radius in the region where the $\mathrm{X}$-ray halo emits.

\section{The isolated ellipticals NGC 7052 and NGC 7785}

The two elliptical galaxies we study here are members of a sample of 43 bright isolated galaxies (Focardi \& Kelm, in prep.) selected from the Updated Zwicky Catalog (UZC, Falco et al. 1999) by means of an adapted version of the Focardi \& Kelm (2002) neighbor-search code. Seven of those are early-type objects, for four of them results of the X-ray analysis of proprietary and archival data have been published by Memola et al. (2009), and finally, for two of them (NGC 7052 and NGC 7785) the available X-ray statistics allows us to derive the dark matter content and distribution.

We recall the criteria to select the sample of truly isolated galaxies: $a$ ) absolute $B$ luminosity higher than $1.3 \times 10^{10} h_{75}^{-2} L_{B \odot}$, where $L_{B_{\odot}}$ is the $B$-band solar luminosity; $b$ ) recessional velocity in the range [2500-5000] $\left.\mathrm{km} \mathrm{s}^{-1} ; c\right)$ galactic latitude $\left(b^{\mathrm{II} \mid} \geq 15^{\circ}\right)$; and $d$ ) no companion galaxies down to 2 mag fainter than the target galaxy within a circular radius of $1.3 h_{75}^{-1} \mathrm{Mpc}$ and at velocity distance of $1000 \mathrm{~km} \mathrm{~s}^{-1}$. These ellipticals are selected in an objective way and their isolation is granted at a typical cluster/group scale. Moreover, NGC 7785 is present in the AMIGA database of isolated galaxies (Verdes-Montenegro et al. 2005) and is also part of the Smith et al. (2004) sample.
Table 1. Physical properties of the galaxies.

\begin{tabular}{ccccccc}
\hline \hline $\begin{array}{c}\text { Source } \\
(1)\end{array}$ & $\begin{array}{c}r_{\mathrm{c}} \\
(2)\end{array}$ & $\begin{array}{c}\beta \\
(3)\end{array}$ & $\begin{array}{c}M_{B} \\
(4)\end{array}$ & $\begin{array}{c}m_{B_{\mathrm{TC}}} \\
(5)\end{array}$ & $\begin{array}{c}K \\
(6)\end{array}$ & $\begin{array}{c}\mathrm{kpc} /{ }^{\prime \prime} \\
(7)\end{array}$ \\
\hline NGC 7052 & $1.11^{\prime \prime}$ & 0.48 & -21.46 & 12.73 & 8.57 & 0.323 \\
NGC 7785 & $35^{\prime \prime}$ & 0.95 & -21.38 & 12.34 & 8.45 & 0.247 \\
\hline
\end{tabular}

Notes. Columns: (1) galaxy name; (2) core radius of the best-fit $\beta$-model; (3) slope $\beta$; (4) absolute $B$-band magnitude; (5) $B$-band magnitude; (6) $K_{\mathrm{s}}$-band magnitude, 2MASS total magnitude; (7) scale in kpc/", which also provides the distance assumed. $B$ - and $K_{\mathrm{s}}$-band magnitudes are from LEDA or NED.

The importance of selecting objects in total isolation was discussed in the introduction: dark matter halos of cluster ellipticals merge with the cluster halo at a scale of $\sim 300 \mathrm{kpc}$, a scale comparable with that of their virial radii. This complicates the dynamical analysis, and more importantly, it implies that the present-day halos around cluster galaxies are not the cosmological dark matter halos anymore, indeed, ellipticals at the center of clusters are found to have more DM than ellipticals in lowdensity regions (Nagino \& Matsushita 2009). The very low density of the environment of isolated ellipticals makes them precious laboratories for investigating the cosmological properties of DM halos around early-type galaxies.

Note that NGC 7052 and NGC 7785, with $L_{X}<2 \times$ $10^{41} \mathrm{erg} \mathrm{s}^{-1}$, and $M_{\mathrm{TOT}}<2 \times 10^{12} M_{\odot}$ (see Table 3 in Memola et al. 2009) do not appear to be fossil groups (e.g., Jones et al. 2003; Pompei et al. 2007) because they do not show their physical properties. Fossil groups (e.g., NGC 1132, Mulchaey \& Zabludoff 1999) have, indeed, so far been observed with larger masses $10^{13}-10^{14} M_{\odot}$, and X-ray luminosity $>10^{42} \mathrm{erg} \mathrm{s}^{-1}$ (see Fig. 8 in Memola et al. 2009).

\section{X-ray observations}

\subsection{X-ray data}

NGC 7052 and NGC 7785 have extended X-ray halos out to 16 and $32 \mathrm{kpc}$, respectively, with hot gaseous mass of $M_{\mathrm{gas}}=$ $2.2 \times 10^{9} M_{\odot}$, and $M_{\text {gas }}=4.6 \times 10^{9} M_{\odot}$. Chandra observations of NGC 7052 and XMM-Newton observations of NGC 7785 have been presented and discussed in Memola et al. (2009). NGC 7052 was observed by the Chandra ACIS-S instrument in September 2002 for about $10 \mathrm{ks}$, and NGC 7785 was observed by the XMM-Newton EPIC detectors in June 2004 for about $15 \mathrm{ks}$ (PrimeFullWIndow mode and thin filter). For the spectral analysis of NGC 7052 we refer to Memola et al. (2009).

The net radial surface brightness profiles, centered at the $\mathrm{X}$-ray peak of the EPIC-pn data in the 0.5-2.0 keV energy band, are well reproduced by a $\beta$-model whose best-fit values of $r_{\mathrm{c}}$ and $\beta$ are given in Table 1 (see also Memola et al. 2009).

\subsection{The X-ray spectral analysis}

We present another X-ray spectral analysis of the NGC 7785 data. Thanks to the quality of the X-ray data and in view of the structure of Eq. (1), we aim to deeply investigate the temperature profile of the X-ray gas emitting component of this isolated elliptical galaxy.

Source counts were extracted for the XMM-Newton EPICMOS1, -MOS2, and -pn from two separate, but contiguous, regions: a $65^{\prime \prime}$ radius circle centered on the source, and a concentric annulus with $65^{\prime \prime}$ and $130^{\prime \prime}$ radii. The background spectrum 

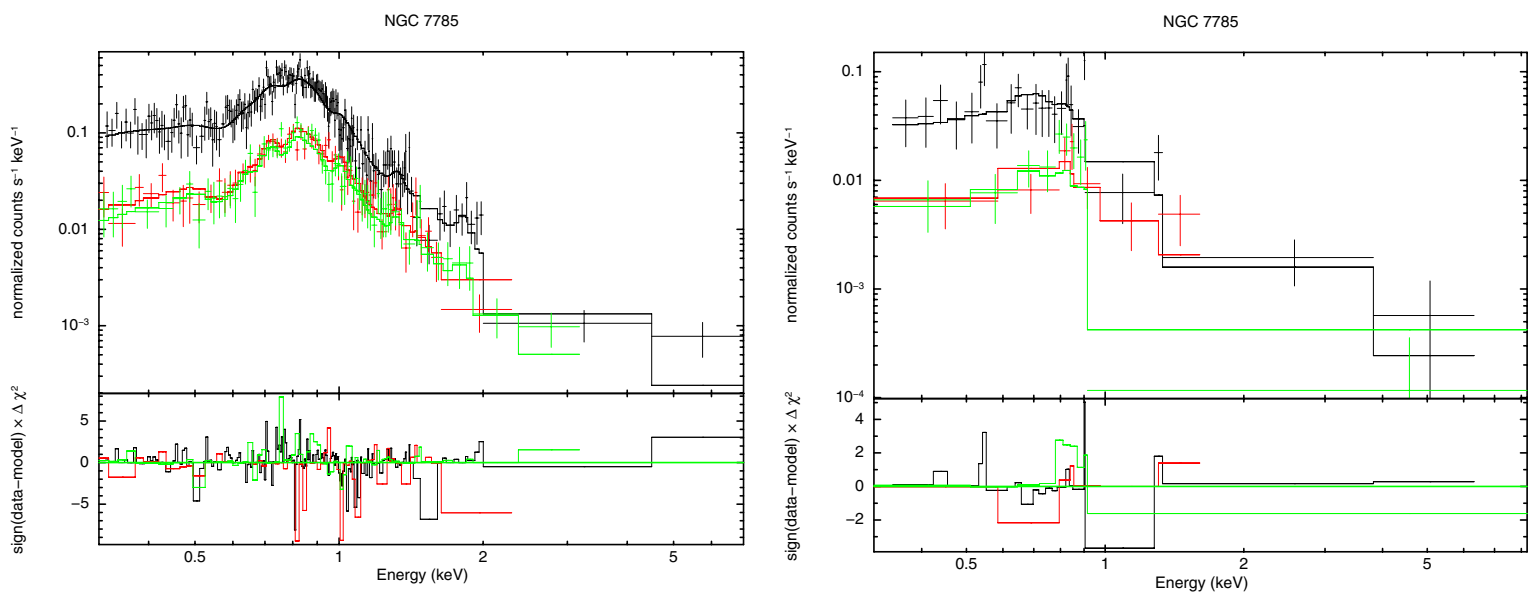

Fig. 1. XMM-Newton EPIC-MOS1 (red), -MOS2 (green), and -pn (black) spectrum and $\chi^{2}$ behavior for NGC 7785. Left panel: extraction radius of $65^{\prime \prime}$. Right panel: extraction annulus with $65^{\prime \prime}$ and 130 " radii. The best-fit model (solid line) is a power-law plus a thermal component (see color figure online).

Table 2. NGC 7785: summary of the XMM-Newton spectral results.

\begin{tabular}{lccccccc}
\hline \hline $\begin{array}{l}N_{\mathrm{H}_{\mathrm{gal}}} \\
\mathrm{cm}^{-2}\end{array}$ & $\begin{array}{c}\text { Region } \\
\prime \prime\end{array}$ & $\begin{array}{c}\text { cts } \\
\text { pn }\end{array}$ & $\begin{array}{c}k T_{\text {mekal }} \\
\mathrm{keV}\end{array}$ & $\begin{array}{c}\Gamma \\
\text { fixed }\end{array}$ & $\chi^{2} /$ d.o.f. & $\begin{array}{c}L_{\text {mekal(0.5-2.0) }} \\
\mathrm{erg} \mathrm{s}^{-1}\end{array}$ & $\begin{array}{c}L_{\mathrm{po}(2.0-10)} \\
\mathrm{erg} \mathrm{s}^{-1}\end{array}$ \\
\hline $5.24 \times 10^{20}$ & 65 & $2687 \pm 59$ & $0.57_{-0.02}^{+0.01}$ & 2.0 & $307 / 314$ & $6.3 \times 10^{40}$ & $6.9 \times 10^{39}$ \\
$5.24 \times 10^{20}$ & $65-130$ & $615 \pm 64$ & $0.32_{-0.05}^{+0.09}$ & 2.0 & $39 / 39$ & $9.4 \times 10^{39}$ & $5.4 \times 10^{39}$ \\
\hline
\end{tabular}

Notes. The EPIC-pn net counts are derived from the regions (a circle and an annulus) of radii given in the second column.

was extracted from a circular source-free region of $90^{\prime \prime}$, at 3.8 arcmin distance from the target. The X-ray spectra were analyzed with the XSPEC package (version 12.6.0; Arnaud 1996). The source counts were stacked into energy bins such that each bin has a significance of at least $2 \sigma$, after background subtraction. The quoted errors on the best-fit parameters correspond to the $90 \%$ confidence level for one interesting parameter (i.e., $\Delta \chi^{2}=2.71$; Avni 1976).

In both cases the best-fit function of the source counts to fit the hot gas includes a power-law with index fixed at $\Gamma=2$, which models the central X-ray binaries or AGN component, plus a thermal (mekal model) component with abundances fixed at 50\% the solar values. For both components the appropriate Galactic hydrogen column density $\left(N_{\mathrm{H}}\right)$ along the line of sight (Dickey \& Lockman 1990) has been taken into account. We found (see Fig. 1 and Table 2) $k T_{\mathrm{g}}=0.32_{-0.05}^{+0.09} \mathrm{keV}$ in the outer annulus and $k T_{\mathrm{g}}=0.57_{-0.02}^{+0.01} \mathrm{keV}$ in the inner circle, in agreement with Memola et al. (2009). Hereafter, we assume $T_{\text {mekal }}=T_{\mathrm{g}}$.

A best-fit for the outer region $\left(\chi^{2} /\right.$ d.o.f. $\left.=39 / 39\right)$ with two free parameters gives the $k T=0.32_{-0.05}^{+0.0} \mathrm{keV}$ value, which is incompatible with $k T=0.56 \pm 0.02 \mathrm{keV}$ that refers to the hypotesis of constant temperature. Our result is fairly robust: a constant value of $k T_{\mathrm{g}}$ out to $130 \mathrm{arcsec}$ can be excluded at $>4 \sigma$ level, and consequently a temperature gradient emerges that we fit linearly.

In detail, the data are well reproduced by

$k T_{\mathrm{g}}(r)=\left[0.6-1.2 \times 10^{-2}(r-3)\right] \mathrm{keV}$,

with $r$ in kpc. This result is not surprising and agrees with the general behavior of isolated ellipticals: Nagino \& Matsushita (2009) find that these objects tend to show a negative temperature gradient. Notice that this variation implies in Eq. (1) a mild contribution from the temperature gradient term that it is smaller than the one from the gas density gradient. Therefore, the effect of the uncertainty in the temperature profile on the mass distribution of Eq. (4) is negligible.

In NGC 7052 we cannot statistically support any evidence of gas temperature variation. For this galaxy, then, $\mathrm{d} T / \mathrm{d} r=0$ as claimed in Memola et al. (2009). A constant temperature has also been observed in some other isolated ellipticals, e.g. NGC 57, NGC 7796, IC 1531 (O'Sullivan et al. 2007).

\section{Structural parameters of the stellar spheroids}

NGC 7785 is known to display boxy isophotes (Lauer 1985), and taking this into account, we re-derive its structural parameters from the $K_{\mathrm{s}}$-band image (see Fig. 2) taken from the Two Micron All Sky Survey (2MASS) atlas. The pixel size is 1 arcsec, and the point-spread function full-width half maximum is 2.9 arcsec. We used the two-dimensional galaxy fitting algorithm GALFIT (Peng et al. 2002) for fitting one Sérsic component, which is allowed to have disky or boxy isophotes. The sky value is fixed to the value provided in the 2MASS image header. The half-light radius is found to be $r_{\mathrm{e}}=22.70^{\prime \prime}$ and the Sérsic index $n=3.8$, very close to the previously found de Vaucouleurs fit $r_{\mathrm{e}}=19.5^{\prime \prime}$ (Bender et al. 1988). The GALFIT boxiness parameter for the best-fit model is $C_{0}=0.19$, and we verified that the addition of this parameter in the case of this galaxy does not change the halflight radius or the Sèrsic index. If, instead of fixing the sky value, we let it be a free parameter, which is determined by GALFIT, both the $r_{\mathrm{e}}$ and $n$ values are 10\% lower. This effectively sets the uncertainty of the fit, which is negligible for the results in the succeeding sections of this work. The performance of this fit can be seen from the residual image in Fig. 2c.

We also compared the surface brightness profile from the above fit with the surface brightness profile of the galaxy image 

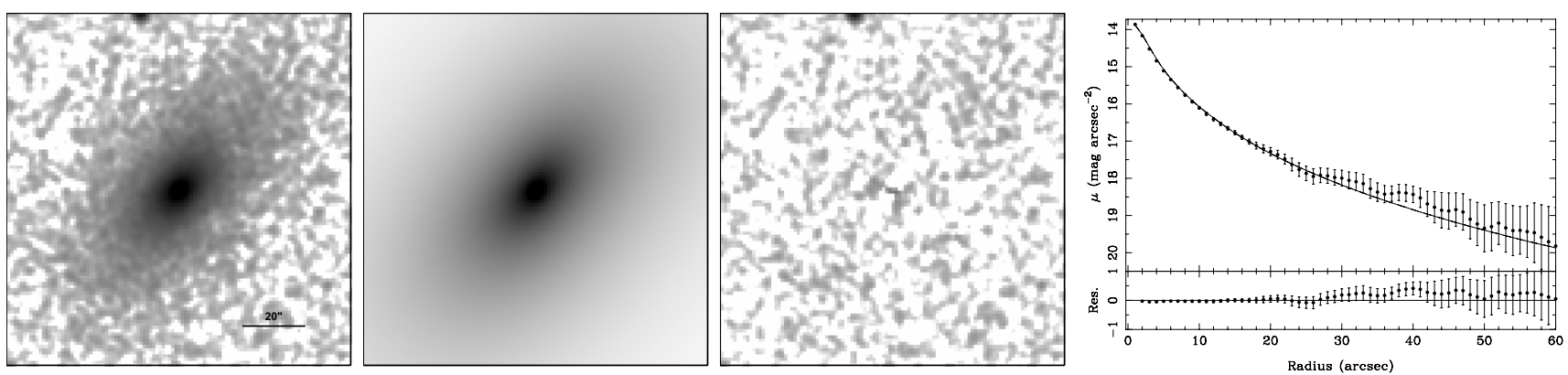

Fig. 2. Two-dimensional 2MASS $K_{\mathrm{s}}$-band image decomposition of NGC 7785. From left to right: the original image, the model image, the residual image, and the surface brightness profile plot. The image surface brightness profile is marked by filled circles and the model with a solid line. The model-data residuals are also shown.

constructed by means of the IRAF task ELLIPSE. The result is shown in Fig. 2.

In NGC 7052 the photometry is simple and well studied by Bender et al. (1988): the distribution of the stars is well described by the usual de Vaucouleurs spheroid with half light radius of $r_{\mathrm{e}}=24^{\prime \prime}$. We confirmed this value with a two-dimensional image decomposition of a 2MASS $K_{\mathrm{s}}$-band image performed as above. However, we omit the plots for brevity reasons.

\section{Stellar spheroid mass in NGC 7052 and NGC 7785}

It is crucial to obtain an independent estimate of the mass of the stellar spheroid for these objects. We computed them by fitting the observed spectral energy distribution (SED) with synthetic spectra. We proceeded as follows: photometric data in $B, V$, and 2MASS $J, H, K_{\mathrm{S}}$ bands were adopted from NED (homogenized NED values) and corrected for Galactic extinction from Schlegel et al. (1998). We used the total magnitudes in 2MASS bands, which are obtained by integrating the radial surface brightness profile out to $r_{\text {tot }}=87.1^{\prime \prime}$ and 82.2" for NGC 7052 and NGC 7785, respectively. Given that half-light radii for these two galaxies are $r_{\mathrm{e}}=24^{\prime \prime}$ and 22.7", integration out to nearly four times these radii provides a good estimate of the total galaxy magnitude (Jarrett et al. 2000). The error estimate for the total magnitude is 0.02 mag for both galaxies. The uncertainty introduced by the extinction correction and possible systematic differences between bands is accounted for by considering an additional $0.05 \mathrm{mag}$ error in quadrature. Photometric data for NGC 7785 were available in the above five bands, while for NGC 7052 the $V$-band data were unavailable.

The stellar mass is obtained from the mass-to-light ratio of the best-fitting model and the $K_{\mathrm{S}}$ values (see Table 1). We fit the synthetic spectra to the observed spectral energy distribution by using the modified version hyperzmass of the HyperZ code (Bolzonella et al. 2000), kindly provided by Bolzonella. The redshifts are fixed to $z=0.0156$ and $z=0.0127$, for NGC 7052 and NGC 7785, respectively. The adopted set of synthetic spectra consists of composite stellar populations with two bursts of star formation, based on simple stellar population models of Maraston (2005) and constructed using the code GALAXEV (Bruzual \& Charlot 2003). The Salpeter initial mass function and solar metallicity are adopted. The strength of the more recent burst is 5\% of that of the older one, and they are separated by a period varying in the range $0-11$ Gyr. The adopted starformation rate is consistent with what is typically found for elliptical galaxies and includes an old major burst at approximately the halo-formation redshift and the presence of a weaker burst in the last several gigayears.
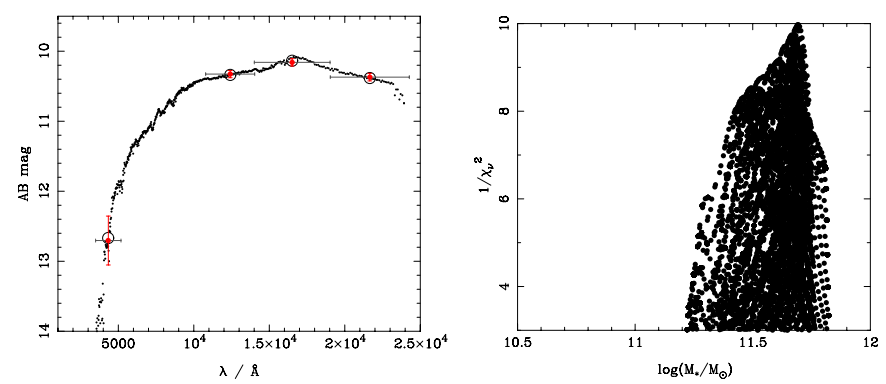

Fig. 3. NGC 7052. Left: model fit to photometry (see color figure online). The best-fit spectral template is marked with points, the corresponding model magnitudes in the observed bands with circles. The observed magnitudes are indicated with filled red circles and error bars. The (black) bars in the wavelength direction denote the bandwidth of respective filters. Right: the likelihood of the stellar mass estimates.
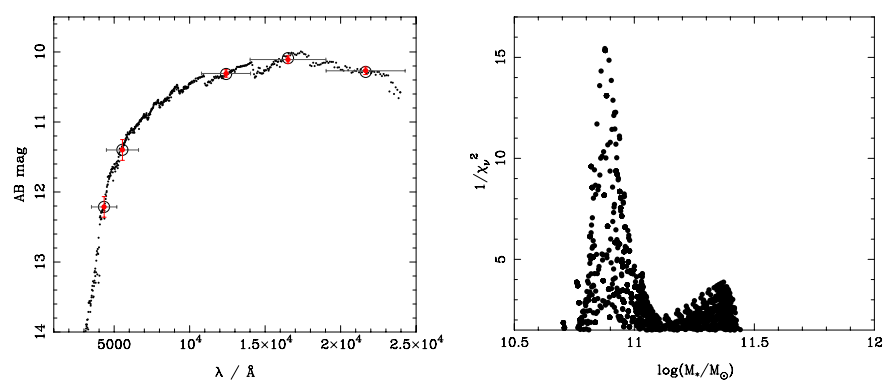

Fig. 4. Same as Fig. 3 for NGC 7785.

For NGC 7052 the best-fit model (Fig. 3, filled black circles) reproduces the data well (model magnitudes: open circles; data: filled red circles and error bars). It consists of two bursts of star formation, one $11.3 \mathrm{Gyr}$ ago, and the second one, of relative strength 5\%, still occurring. The resulting stellar spheroid spectrophotometric mass is $\log \left(M_{\mathrm{sph}}^{\mathrm{s}} / M_{\odot}\right)=11.6_{-0.2}^{+0.1}$, at $2 \sigma$ fitting uncertainty, then $\log \left(M_{\mathrm{sph}}^{\mathrm{s}} / L_{B}\right)=0.84_{-0.2}^{+0.1}$.

For NGC 7785 the best-fit model reproduces the data well, in this case it points to a main burst $9.0 \mathrm{Gyr}$ ago, followed by the second weak one $8.0 \mathrm{Gyr}$ ago. For this object, a single burst of star formation about $9.0 \mathrm{Gyr}$ ago would also be a satisfactory model. The stellar spheroid spectrophotometric mass is found to be $\log \left(M_{\mathrm{sph}}^{\mathrm{s}} / M_{\odot}\right)=10.9_{-0.1}^{+0.3}$, at $2 \sigma$ fitting uncertainty, then $\log \left(M_{\mathrm{sph}}^{\mathrm{s}} / L_{B}\right)=0.20_{-0.1}^{+0.3}$.

Figures 3 and 4 show the spectral template fitting (left) and the mass determination (right) for NGC 7052 and NGC 7785, respectively. In both cases a large number of different SFRs yield a stellar mass similar to the one we assume. Models in the likelihood plot differ by the age of the two components and the 

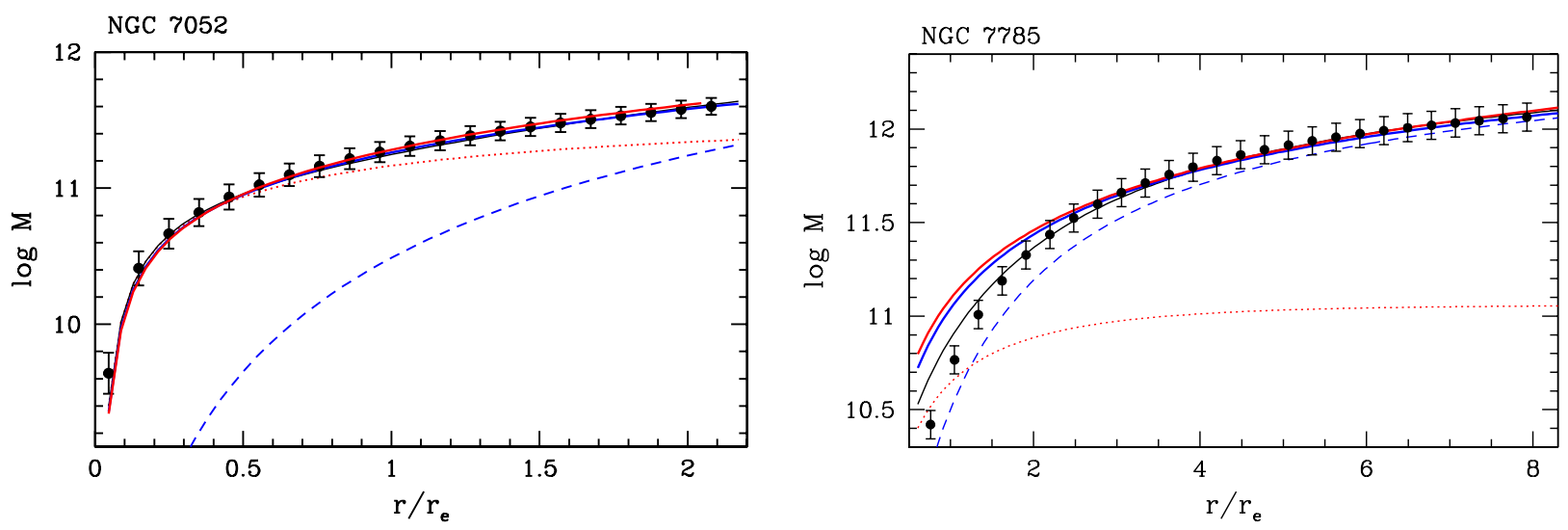

Fig. 5. Left: mass models of NGC 7052. The X-ray derived dynamical mass (filled circles with error bars) is modeled (thin black line) including a stellar spheroid (dotted line) and a Burkert halo (dashed line). Also shown is the best-fit solution for the total mass for models that assume a NFW profile to represent the dark halo (blue line) or the MOND framework (red solid lines). $r_{\mathrm{e}}$ corresponds to $7.7 \mathrm{kpc}$. Right: mass models of NGC 7785. $r_{\mathrm{e}}$ corresponds to $5.6 \mathrm{kpc}$ (see color figure online).

Table 3. Structural properties.

\begin{tabular}{cccccccccccccc}
\hline \hline $\begin{array}{c}\text { Source } \\
(1)\end{array}$ & $\begin{array}{c}\mathrm{lpt} \\
(2)\end{array}$ & $\begin{array}{c}r_{\mathrm{e}} \\
(3)\end{array}$ & $\begin{array}{c}n \\
(4)\end{array}$ & $\begin{array}{c}M_{\mathrm{lpt}} \\
(5)\end{array}$ & $\begin{array}{c}M_{\mathrm{sph}} \\
(6)\end{array}$ & $\begin{array}{c}M_{\mathrm{sph}} / L_{B} \\
(7)\end{array}$ & $\begin{array}{c}r_{0} \\
(8)\end{array}$ & $\begin{array}{c}\rho_{0} \\
(9)\end{array}$ & $\begin{array}{c}M_{\mathrm{vir}} \\
(10)\end{array}$ & $\begin{array}{c}c \\
(11)\end{array}$ & $\begin{array}{c}M_{\mathrm{vNFW}} \\
(12)\end{array}$ & $\begin{array}{c}R_{\mathrm{vir}} \\
(13)\end{array}$ & $\begin{array}{c}f_{\mathrm{baryon}} \\
(14)\end{array}$ \\
\hline NGC 7052 & 16.1 & 7.7 & 4 & 4.0 & 3.6 & 6.3 & 22.3 & 1.3 & 7.4 & 10 & 8.6 & 476 & 0.05 \\
NGC 7785 & 32.1 & 5.6 & 3.8 & 9.0 & 1.1 & 2.3 & 8.7 & 10.2 & 3.6 & 33 & 5.2 & 405 & 0.03 \\
\hline
\end{tabular}

Notes. Columns: (1) galaxy name; (2) last measured point, in arcsec; (3) half-light radius in kpc; (4) Sérsic index; (5) mass inside the last measured point, in units of $10^{11} M_{\odot} ;(6)$ spheroid mass in units of $10^{11} M_{\odot}$; (7) mass-to-light ratio of the spheroid in $B$-band, in units of $M_{\odot} / L_{B_{\odot}} ;(8)$ dark matter Burkert halo core radius in kpc; (9) dark matter Burkert halo central density in units of $10^{-24} \mathrm{~g} \mathrm{~cm}^{-3}$; (10) Burkert halo virial mass in units of $10^{12} M_{\odot} ;(11)$ concentration parameter $c$ of NFW halo; (12) NFW halo virial mass in units of $10^{12} M_{\odot}$; (13) Burkert halo virial radius in kpc; (14) baryonic fraction.

reddening, which is for simplicity assumed to be the same for both components. The mass estimates obtained by this method are very robust: they primarily depend on the normalization of the SED in the near-infrared bands, and are relatively insensitive to the details of the star-formation history (Maraston et al. 2010). In particular, changing the star-formation history to a single starformation episode of varying length and functional form results in a maximum $10 \%$ change in the stellar mass (for the best-fit model) for NGC 7785 and $20 \%$ for NGC 7052; varying metallicity in the range between half-solar and two solar metallicity leaves the mass unchanged for NGC 7785 and 30\% smaller for NGC 7052; changing the relative strength of the second burst from $5 \%$ to $10 \%$ gives the same mass for NGC 7785 and a $10 \%$ lower mass for NGC 7052. Again, these modifications mainly influence the implied age for the population, with a small effect on the deduced stellar mass, which is well within the quoted error bars. Using the Chabrier or Kroupa initial mass function instead of the Salpeter one will result in mass-to-light ratios $\approx 1.7$ times lower (Maraston 2005).

\section{Mass models}

We fitted the profile of the gravitating mass $M(r)$ obtained by Eq. (1) with a mass model $M_{\text {model }}(r)$ that includes a luminous spheroid and a dark halo (Bertola et al. 1993; Kronawitter et al. 2000):

$M_{\text {model }}(r)=M_{\star}(r)+M_{\mathrm{h}}(r)$.

In these objects the stellar surface brightness follows a Sérsic profile with index $n$ and effective radii $r_{\mathrm{e}}$ given in Table 3. Once we deproject $r_{\mathrm{e}}$ under the reasonable assumptions of spherical symmetry and constant stellar mass-to-light ratio, we obtain the spatial density $\rho_{\star}(r)$ and mass profile $M_{\star}(r)=\int_{0}^{r} 4 \pi r^{\prime 2} \rho_{\star}\left(r^{\prime}\right) \mathrm{d} r^{\prime}$ of the stellar component.

Let us note that in NGC 7052 the X-ray mass measurement extends (only) to $16 \mathrm{kpc}$, corresponding to $\simeq 2 r_{\mathrm{e}}$. Not surprisingly, in this object the dynamical mass matches the spheroid mass over most of the probed region, and the contribution of the $\mathrm{DM}$ to the gravitating matter is small overall. Thus, to derive the structural parameters of the former component from the latter leads to results with sizable uncertainties.

We assume as in spirals that the dark matter is distributed in a spherical halo with a Burkert density profile (Salucci \& Burkert 2000):

$\rho(r)=\frac{\rho_{0} r_{0}^{3}}{\left(r+r_{0}\right)\left(r^{2}+r_{0}^{2}\right)}$,

where the core radius $r_{0}$ and the central density $\rho_{0}$ are free parameters. The dark mass profile $M_{\mathrm{h}}(<r)$ takes the form:

$M_{\mathrm{h}}(r)=M_{0}\left\{\ln \left(1+\frac{r}{r_{0}}\right)-\tan ^{-1}\left(\frac{r}{r_{0}}\right)+\frac{1}{2} \ln \left[1+\left(\frac{r}{r_{0}}\right)^{2}\right]\right\}$,

where $M_{0} \equiv 6.4 \rho_{0} r_{0}^{3}$.

Ellipticals comply with the inner baryon dominance paradigm like spirals (van Albada et al. 1985; Salucci \& Persic 1999).

The mass profile $M(r)$ obtained from the above equations, with $M_{\text {model }}\left(r, M_{\star}, \rho_{0}, r_{0}\right)$ given by Eq. (6) for our two objects is fitted by minimizing the $\chi^{2}$. Estimated uncertainties are 
$\delta \log M=0.075$ dex and 0.06 dex for NGC 7052 and NGC 7785, respectively. The results are shown in Fig. 5; the structural galaxy parameters, i.e., the spheroid mass, the mass, the core radius, and the central density of the halo are given in Table 3.

The mass model with a Burkert halo and a Sérsic spheroid gives a good fit to the distribution of the gravitating mass of our galaxies: almost all data are reproduced within the $1 \sigma$ error bars and $\chi_{\text {reduced }}^{2} \sim 1$. The transition region between the region dominated by the stellar spheroid and the region dominated by the dark matter occurs at $(2.0 \pm 0.3) r_{\mathrm{e}}$. In both galaxies, we find that the stellar baryonic component dominates the gravitational potential inside $r_{\mathrm{e}}$, in agreement with Bertola et al. (1993), and, e.g., Fukazawa et al. (2006). At $r_{\mathrm{e}}$, the fraction of the gravitating mass contained in the stellar spheroid amounts to $(76 \pm 5) \%$ in NGC 7052, and $\left(84_{-15}^{+5}\right) \%$ in NGC 7785 where the lower spatial resolution makes the estimate more difficult. Then, the $(M / L)$ profiles at small radii match those expected from the stellar component alone.

We find that the stellar spheroid masses are $M_{\mathrm{sph}}=1.1_{-0.3}^{+0.4} \times$ $10^{11} M_{\odot}$ in NGC 7785 , and $M_{\text {sph }}=3.6_{-1.0}^{+1.3} \times 10^{11} M_{\odot}$ in NGC 7052, where the quoted uncertainties are the fitting ones. The stellar mass fraction inside $r_{\mathrm{e}}$ found here for both galaxies is quite consistent with earlier works (e.g., Gerhard et al. 2001; Thomas et al. 2009; and several recent SLAC papers starting with Koopmans et al. 2006). For NGC 7052 one by-product of the good agreement we find between the X-ray mass profile and the stellar one indicates that in this system there are no strong deviations from the hydrostatic equilibrium condition, which may lead to an underestimate of the cumulative mass, in agreement with previous studies (Johnson et al. 2009).

In any case, we find that in NGC 7052 the X-ray derived mass profile is well fitted by the mass models. This is not obvious and in turn indicates that these measurements are very reliable. Indeed, between $0.3 r_{\mathrm{e}}$ and $0.8 r_{\mathrm{e}}$ the mass profile of a de Vaucoulers spheroid has a unique distinct feature that we find in the data.

Remarkably, these values agree very well with the above SED fitting estimates. Mass modeling leads in the $B$-band to a spheroid mass-to-light ratio of $M_{\mathrm{sph}} / L_{B}=6.3 M_{\odot} / L_{B_{\odot}}$ for NGC 7052, and $M_{\mathrm{sph}} / L_{B}=2.3 M_{\odot} / L_{B_{\odot}}$ for NGC 7785 , with a fitting uncertainty of $30 \%$ (see Table 3 ). Note that the value for NGC 7785 is on the lower side for ellipticals, but it is confirmed by spectral fitting. In fact, in NGC 7785 the spectrophotometric estimate is $M_{\mathrm{sph}}^{\mathrm{s}} / L_{B}=1.7 M_{\odot} / L_{B_{\odot}}\left(M_{\mathrm{sph}}^{\mathrm{s}}=8 \times 10^{10} M_{\odot}\right)$, while in NGC 7052 it is $M_{\mathrm{sph}}^{\mathrm{s}} / L_{B}=6.9 M_{\odot} / L_{B_{\odot}}\left(M_{\mathrm{sph}}^{\mathrm{s}}=4 \times 10^{11} M_{\odot}\right)$.

Before we discuss the DM properties, notice that in NGC 7052 the dark matter dominates the mass distribution only at the outermost radii, for which we have X-ray data $\left(1.5 r_{\mathrm{e}}<\right.$ $\left.r<2.0 r_{\mathrm{e}}\right)$, which makes the estimate of its structural parameters uncertain.

The dark matter halos show a cored density distribution. The core radii and the corresponding central densities take in NGC 7052 and NGC 7785 the values of $22.3 / 8.7 \mathrm{kpc}$ and $1.3 \times 10^{-24} / 1.0 \times 10^{-23} \mathrm{~g} \mathrm{~cm}^{-3}$. However, these estimates are uncertain: even considering the mass profiles we infer from $\mathrm{X}$-ray fluxes as error-free, the fitting uncertainties on these two parameters would be at the level of $50 \%$. Furthermore, unlike for spirals where the dynamical mass is quite well determined from the rotation curve, here there is a non-negligible uncertainty in the (derived) mass profile that we fitted with the model $\delta \mathrm{d} \log M(r) / \mathrm{d} \log r \simeq 0.15$, which contributes an additional uncertainty of $\sim 30 \%$ on the derived values of $r_{0}$ and $\rho_{0}$. The comparison of these values with those in spirals of the same mass must await more and more precise mass models; however, we can already claim that DM halo around ellipticals seem to be denser and with a smaller core radius, but the product $\rho_{0} r_{0}$ is still, approximately, the same as the one found in smaller objects and for different Hubble types (Donato et al. 2009).

Notice that in NGC 7785, similarly to what it is found in (some) other ellipticals, the X-ray derived mass profile in the very inner galaxy region, $r<r_{\mathrm{e}}<6 \mathrm{kpc}$, corresponding to the innermost 2-3 data points, cannot be reproduced by any reasonable mass profile, including a Sersic spheroid, a standard DM halo or a combination of both. The dynamical X-ray mass appears, for $r \rightarrow 0$, to be progressively smaller than the actual data. This discrepancy, probably caused by the contribution to the X-ray emission of i) non-thermal pressures or ii) multipletemperature components (see Das et al. 2010) does not affect the main results of the mass modeling, however. Indeed in the Burkert class spheroidal (BS) model the spheroid mass has been obtained by imposing that at $2 r_{\mathrm{e}}$, i.e., at a radius outside the troubled region, the spheroid and the dark halo masses equal the dynamical mass.

Remarkably, we note that the resulting value for the spheroid mass is consistent with the photometric estimates.

Finally, we checked the effects on the resulting best fitting values of the DM halo structural parameters $\left(\rho_{0}\right.$ and $\left.r_{0}\right)$ of 1$)$ a variation of the spheroid mass of $\pm 30 \% ; 2$ ) by considering the latter quantity as a free parameter; 3 ) including the two innermost data points. In all these checks, we found that the BS model fits the dynamical mass very well and the resulting values of $\rho_{0}$ and $r_{0}$ lie always within $30 \%$ those given in Table 3 . The presence of a cored DM mass distribution relies on the fact that between $r_{\mathrm{e}}$ and $3 r_{\mathrm{e}}$, i.e., well outside the troubled region, the dynamical mass shows an increase steeper than linear.

We can estimate the virial masses and radii for NGC 7052 and NGC 7785 by extrapolating the mass models: $M_{\mathrm{vir}} \simeq$ $8.6 \times 10^{12} M_{\odot}, M_{\text {vir }} \simeq 5.2 \times 10^{12} M_{\odot}, R_{\text {vir }}=476 \mathrm{kpc}$, and $R_{\text {vir }}=405 \mathrm{kpc}$. Indeed, in the present case the extrapolation of the outermost X-ray mass $M\left(R_{\mathrm{o}}\right)$ to the virial radius is feasible. The X-ray mass profiles indicate that the present data have reached, contrary to the "extended" available HI RCs in spirals, the region in which the circular velocity $\propto(M / R)^{0.5}$ has a maximum (Salucci et al. 2007).

We are allowed to extrapolate $M(r)$ because we know from weak-lensing measurements that DM halos around isolated galaxies are not truncated out to their virial radii (Mandelbaum et al. 2006) and that their outer profiles are consistent with a Burkert/NFW profile. The extrapolation uncertainties are at the level of $15 \%$ for NGC 7785, and $20 \%$ for NGC 7052. The uncertainty on $M\left(R_{\mathrm{o}}\right) \propto \beta T\left(R_{\mathrm{o}}\right) \beta r_{\mathrm{c}}^{-1}$ is also small, because the observational uncertainties on $T\left(R_{\mathrm{o}}\right), \beta, r_{\mathrm{c}}$ are at a level of $10 \%$ (Memola et al. 2009).

These estimates of the virial mass from direct mass measurements are solid: these are two of the few cases in which this has been done so far.

This confirms that big ellipticals live in halos two to four times more massive than those around big spirals.

The baryon fractions, i.e., the ratios of stellar and virial mass, are $0.05 \pm 0.02$ and $0.03 \pm 0.01$, where the main uncertainty comes from how we extrapolate the dark mass profile out to the virial radius. The values found agree with the determinations of Shankar et al. (2006) obtained by correlating the baryonic mass function with the halo mass function.

In summary, the fractional amount of the stellar component looks (within a factor of two) similar to that found in the biggest spirals (Salucci et al. 2007) and is in any case much smaller than 
the cosmological ratio of $\sim 1 / 7$. Given the large gravitational potential well of these galaxies, it remains to be understood where $70-80 \%$ of the original cosmological baryons went. The dark matter mass profile in the central parts indicates a cored density distribution, although the estimate of the characterizing structural parameters (central density and core radius) is uncertain.

\subsection{NFW halo profile}

We further investigated the halo mass profile by fitting the dynamical mass profiles with the NFW halo profile that emerged in the $\Lambda$ CDM scenario (Navarro et al. 1997):

$M_{\mathrm{NFW}}(r)=M_{\mathrm{vir}} \frac{\left[\log \left(1+r / r_{\mathrm{s}}\right)-\left(r / r_{\mathrm{s}}\right) /\left(1+r / r_{\mathrm{s}}\right)\right]}{\log (1+c)-c /(1+c)}$,

with $r_{\mathrm{s}} \equiv R_{\text {vir }} / c$.

We tested this mass distribution, leaving $c$ and $M_{\text {vir }}$ as free parameters, which is necessary to obtain an acceptable value for the reduced $\chi_{\text {red. }}^{2}$. For NGC 7785 we leave the value of the stellar mass unchanged with respect to the one obtained above, because it is irrelevant for the total gravitational potential; for NGC 7052 we leave it free.

The model fits (see the dashed line in Fig. 5) are very satisfactory for NGC $7052\left(\chi_{\text {red }}^{2}<1\right)$ and less acceptable for NGC $7785\left(\chi_{\text {red }}^{2}=3.6\right)$. The resulting NFWS spheroid masses are reasonable and similar to the BS ones. In particular, in NGC 7052 the spheroid mass is $85 \%$ of the one obtained for the Burkert halo mass model.

Moreover, in the latter case the resulting value for the concentration parameter $c=33$ is inconsistent with the $\Lambda$ CDM predictions of $c=8-9$ for objects $\sim 5 \times 10^{12} M_{\odot}$. This is not due to the mass modeling uncertainties, because the gravitating mass inside $30 \mathrm{kpc}>10^{12} M_{\odot}$ is much larger than the spheroid mass, therefore coincides with the halo mass, which implies that this object displays an intrinsically cored mass distribution.

As a counter proof, the standard NFW DM halo of $10^{12} M_{\odot}$ has $c=9$ (e.g., Bertola et al. 1993; Kronawitter et al. 2000; Klypin et al. 2011; Tortora et al. 2010) and it is completely inconsistent with the data.

Note that halo models with $c \simeq 3$, which is equally inconsistent with $\Lambda \mathrm{CDM}$ prediction, but in line with the results of Fukazawa et al. (2006), have a $\chi_{\text {red }}^{2}$ similar to cored best-fit values.

Changing the values of the spheroid masses or considering the adiabatic contraction process for both galaxies for the model with the NFW halo and Sérsic spheroid (NFWS) gives a worse fit. The NFWS model vs observation discrepancy is very different with respect to the BS one. Firstly, it extends outside the (possibly) troubled region, out to $r \leq 2.5 r_{\mathrm{e}} \leq 15 \mathrm{kpc}$. Secondly, the discrepancy is also found in a region where only one component is present, the dark one, which completely dominates the potential with a known density profile. Furthermore, the (very high) value of $c$ does not depend on the innermost values of the dynamical mass, but on the fact that from $r \sim r_{\mathrm{c}} \simeq 1.5 r_{\mathrm{e}} \sim 8 \mathrm{kpc}$ out to the last measured point at $\sim 45 \mathrm{kpc}$ the dynamical mass (completely dominated by the dark component) increases perfectly linearly with the radius. Independently on how we treat the first two data at $r<r_{\mathrm{e}}$, the NFWS mass model for NGC 7785 must have $c \simeq 35$.

\subsection{MOND}

We realize that our objects are in the Mondian regime for $r>5 \mathrm{kpc}$. Our two objects are then well suited for testing the
MOND paradigm, in particular the case in which it is seen as a modification of the inertia law, able to account for the "missing light" in galaxies. In this framework the relation between the density of the gravitating matter and its relative acceleration, when the latter is $\leq a_{0}=1.3 \times 10^{-8} \mathrm{~cm} \mathrm{~s}^{-2}$, is very different from the Galilean-Newtonian case. As a consequence, for a baryonic mass distribution $M_{\mathrm{b}}(r)$, we measure (e.g. by applying the hydrostatic equilibrium condition) a different/larger dynamical mass $M_{\mathrm{MOND}}(r)$ :

$$
M_{\mathrm{MOND}}(r)=\frac{1}{2} M_{\mathrm{b}}(r)\left\{1+\left[1+\frac{4.1 \times 10^{9} M_{\odot}}{M_{\mathrm{b}}(r)}\left(\frac{r}{1 \mathrm{kpc}}\right)^{2}\right]^{0.5}\right\} .
$$

Therefore, we test MOND by fitting $M(r)$ given by Eq. (6) with $M_{\text {MOND }}(r)$, leaving the mass of the spheroid as a free parameter. We can anticipate that MOND will have difficulties accounting for the DM of early-type systems. They have just one baryonic component, the Sérsic spheroid, whose mass converges well inside the region for which we have the dynamical mass. This creates a mass discrepancy that steadily increases with radius as a power-law. In spirals MOND must account for a very similar behavior of the dynamical mass but it has two additional advantages: 1) there are two baryonic components - the stellar and the HI disk, and 2) the mass of the latter does not converge inside the region mapped by dynamical data.

MOND shows a tension in NGC 7052, and fails the test for NGC 7785 (see Fig. 5). The value $\chi_{\text {red }}^{2} \simeq 2$ of this mass model is fairly high. However, even if we consider the MOND mass model satisfactory, the resulting best-fit values for the spheroid mass-to-light ratios $\left(M / L_{B} \simeq 7\right.$ and 15) are inconsistent with the colors of these galaxies.

\section{Conclusions}

We study the mass distribution of two isolated ellipticals by means of their X-ray emission and stellar photometry. We find, and this is one of the few cases at present for early-type galaxies in such suitable environment, that a dark component must be present for $r>r_{\mathrm{e}}$, because $r_{\mathrm{e}}$ is the half-light radius of the stellar spheroid. More specifically, we find that inside $r_{\mathrm{e}}$ the stellar component accounts for $\$ 70 \%$ of the gravitating mass. This result is not new (see, e.g., Kronawitter et al. 2000; Nagino \& Matsushita 2009, where 22 objects with XMM-Newton and Chandra X-ray luminosities are mass-modeled). However, our finding is at odds with the claim of substantial proportions of DM inside $r_{\mathrm{e}}$ (Humphrey et al. 2006) and with the idea that the well known tilt of the fundamental plane of ellipticals is due to systematic variations of the DM fraction inside $r_{\mathrm{e}}$ with galaxy properties. Indeed, our (and other) mass models of ellipticals imply that the central stellar line-of-sight velocity dispersion has very little contribution from the dark component and essentially weighs only the stellar spheroid, whose structural properties (mass-to-light ratio and half light radius) therefore must be considered responsible for the observed tilt.

In our two objects the total mass does not increase with radius as a (unique) power-law, as suggested by Humphrey \& Buote (2010): $\operatorname{dlog} M / \operatorname{dlog} r$ decreases from $\sim 2$ in the innermost regions mapped by X-ray emission $\left(\sim 0.3 r_{\mathrm{e}}\right)$ to $\sim 1$ in the outermost regions $\left(\sim 2 r_{\mathrm{e}}\right)$, a behavior often found in the mass structure of ellipticals (Kronawitter et al. 2000; Fukazawa et al. 2006). The mass profiles of NGC 7052 and NGC 7785 are easily and naturally reproduced by a combination of a Sérsic spheroid and a Burkert halo, where the former is much denser and the latter is much more massive. The fits are good (unfortunately) for a 
large range of values of the structural parameters, i.e., the central DM density, the core radius, and the spheroid mass. This agrees with previous results: ellipticals show a variety of mass profiles, each of them reproducible by a quite large variety of different mass models. Thus, in our objects we find no evidence for the claim that the mass distribution in ellipticals is subject to a "cosmic conspiracy", i.e., that all of them would show the same (unexplained) profile, implying just a relationship rather than the claimed fine-tuning in the DM and luminous matter structural parameters.

As in spirals, NFWS mass models have some difficulties reproducing the data: the best-fitting values of the $\chi^{2}$, the mass to light ratio of the luminous component, the halo concentration parameter are out of the expected range. However, in ellipticals, a NFW profile is not necessarily the correct present-day profile for a $\Lambda C D M$ halo as well. The baryons largely dominate the inner potential and their infall/contraction may have modified the DM distribution (e.g., Klypin et al. 2002).

Finally, our mass determinations are in tension with the baryonic Tully-Fisher relation (McGaugh et al. 2000), according to which in cosmic structures from clusters to dwarf spheroids (dSphs), the circular velocity $V=G M(r) / r^{0.5}$ and the baryonic mass $M_{\mathrm{b}}$ scale as $\log M_{\mathrm{b}}=4 \log V+1.61$. In our objects we obtain a reliable estimate of the stellar mass that coincides with the baryonic one. Moreover, for our objects the mass profile does increase linearly with radius in the outer regions, so that the "circular velocity" is radially constant with radius and can be easily extrapolated out to its virial value. We find that for NGC $7052\left(V, M_{\mathrm{b}}\right)=\left(330 \mathrm{~km} \mathrm{~s}^{-1}, 3.6 \times 10^{11} M_{\odot}\right)$, while for $\operatorname{NGC} 7785\left(V, M_{\mathrm{b}}\right)=\left(380 \mathrm{~km} \mathrm{~s}^{-1}, 1.1 \times 10^{11} M_{\odot}\right)$. These objects have baryonic masses that fall short of the predicted value from this relation by a factor $\sim 2$ and $\sim 8$.

By investigating two isolated ellipticals by means of their $\mathrm{X}$-ray and optical emissions we find that 1) they have a considerable amount of DM, but only at radii $>r_{\mathrm{e}} ; 2$ ) their DM halos have a shallower distribution than that emerging in the $\Lambda$ CDM scenario; 3) these objects are in the Mondian regime, but do not agree with its predictions; 4) these objects do not comply with the baryonic Tully Fisher.

Acknowledgements. We would like to thank C. Maraston and J. Pforr for providing the SED templates and for helpful discussions. We also thank E. Pompei, and an anonymous referee for comments, which improved the manuscript. E.M. thanks L. Ballo, G. Trinchieri, and A. Wolter for priceless scientific hints. This research was performed despite of the financial policy by the Italian government.

\section{References}

Arnaud, K. A. 1996, ASPC, 101, 17

Avni, Y. 1976, 210, 642

Bender, R., Doebereiner, S., \& Moellenhoff, C. 1988, A\&AS, 74, 385

Bertola, F., Pizzella, A., Persic, M., \& Salucci, P. 1993, ApJ, 416, L45

Bolzonella, M., Miralles, J.-M., \& Pelló, R. 2000, A\&A, 363, 476

Bruzual, G., \& Charlot, S. 2003, MNRAS, 344, 1000
Cavaliere, A., \& Fusco-Femiano, R. 1976, A\&A, 9, 137

Das, P., Gerhard, O., Churazov, E., \& Zhuravleva, I. 2010, MNRAS, 409, 1362

Dickey, J. M., \& Lockman, F. J. 1990, ARA\&A, 28, 215

Donato, F., Gentile, G., Salucci, P., et al. 2009, MNRAS, 397, 1169

Ettori, S., \& Fabian A. C. 2006, MNRAS, 369, L42

Fabricant, D., \& Gorestein, P. 1983, ApJ, 267, 535

Falco, E. E., Kurtz, M. J., Geller, M. J., et al. 1999, PASP, 111, 438

Focardi, P., \& Kelm, B. 2002, A\&A, 391, 35

Forman, W., Jones, C., \& Tucker, W. 1985, ApJ, 293, 102

Fukazawa, Y., Botoya-Nonesa, J. G., Pu, J., Ohto, A., \& Kawano, N. 2006, ApJ, 636, 698

Gerhard, O., Kronawitter, A., Saglia, R. P., \& Bender, R. 2001, AJ, 121, 1936

Humphrey, P. J., \& Buote, D. A. 2010, MNRAS, 403, 2143

Humphrey, P. J., Buote, D. A., Gastaldello, F., et al. 2006, ApJ, 646, 899

Jarrett, T. H., Chester, T., Cutri, R., et al. 2000, AJ, 119, 2498

Johnson, R., Chakrabarty, D., O’Sullivan, E., \& Raychaudhury, S. 2009, ApJ, 706, 980

Jones, L. R., Ponman, T. J., Horton, A., et al. 2003, MNRAS, 343, 627

Klypin, A., Zhao, H., \& Somerville, R. S. 2002, ApJ, 573, 597

Klypin, A., Trujillo-Gomez, S., \& Primack, J. 2011, ApJ, accepted [arXiv: 1002 . 3660]

Koopmans, L. V. E., Treu, T., Bolton, A. S., Burles, S., \& Moustakas, L. A. 2006, ApJ, 649, 599

Kormendy, J., \& Freeman, K. C. 2004, in Dark Matter in Galaxies, IAU Symp., 220,377

Kronawitter, A., Saglia, R. P., Gerhard, O., \& Bender, R. 2000, A\&AS, 144, 53

Lauer, T. R. 1985, MNRAS, 216, 429

Mandelbaum, R., Seljak, U., Cool, R. J., et al. 2006, MNRAS, 372, 758

Mandelbaum, R., van de Ven, G., \& Keeton, C. R. 2009, MNRAS, 398, 635

Maraston, C. 2005, MNRAS, 362, 799

Maraston, C., Pforr, J., Renzini, A., et al. 2010, MNRAS, 407, 830

McGaugh, S. S., Schombert, J. M., Bothun, G. D., \& de Blok, W. J. G. 2000, ApJ, 533, L99

Memola, E., Trinchieri, G., Wolter, A., Focardi, P., \& Kelm, B. 2009, A\&A, 497, 359

Mulchaey, J. S., \& Zabludoff A. I. 1999, ApJ, 514, 133

Nagino, R., \& Matsushita, K. 2009, A\&A, 501, 157

Navarro, J. F., Frenk C. S., \& White S. D. M. 1997, ApJ, 490, 493

Oh, S.-H., de Blok, W. J. G., Walter, F., Brinks, E., \& Kennicutt, R. C. 2008, AJ, 136,2761

O’Sullivan, E., Sanderson, A. J. R., \& Ponman, T. J. 2007, MNRAS, 380, 1409

Peng, C. Y., Ho, L. C., Impey, C. D., \& Rix, H.-W. 2002, AJ, 124, 266

Persic, M., Salucci, P., \& Stel, F. 1996, MNRAS, 281, 27

Pompei E., Dahlem, M., \& Iovino A. 2007, A\&A, 473, 399

Pu, S. B., Saglia, R. P., Fabricius, M. H., et al. 2010, A\&A, 516, 4

Romanowsky, A. J. 2010, in Hunting for the Dark: the Hidden Side of Galaxy Formation, Malta, October 2009, ed. V. P. Debattista, \& C. C. Popescu, AIP Conf. Proc., 1240, 351

Salucci, P., \& Burkert, A. 2000, ApJ, 537, L9

Salucci, P., \& Persic, M. 1997, in Dark and Visible Matter in Galaxies, Pusteria, Italy, July 1996, ed. M. Persic, \& P. Salucci, ASP Conf. Ser., 117, 1

Salucci, P., \& Persic, M. 1999, A\&A, 351, 442

Salucci, P., Lapi, A., Tonini, C., et al. 2007, MNRAS, 378, 41

Schlegel, D. J., Finkbeiner, D. P., \& Davis, M. 1998, ApJ, 500, 525

Shankar, F., Lapi, A., Salucci, P., De Zotti, G., \& Danese, L. 2006, ApJ, 643, 14

Smith, R. M., Martínez, V. J., \& Graham M. J. 2004, ApJ, 617, 1017

Thomas, J., Jesseit, R., Saglia, R. P., et al. 2009, MNRAS, 393, 641

Tortora, C., Napolitano, N. R., Romanowsky, A. J., Capaccioli, M., \& Covone, G. 2009, MNRAS, 396, 1132

Tortora, C., Napolitano, N. R., Romanowsky, A. J., \& Jetzer, P. 2010, ApJ, 721, L1

Verdes-Montenegro, L., Sulentic, J., Lisenfeld, U., et al. 2005, A\&A, 436, 443

van Albada, T. S., Bahcall, J. N., Begeman, K., \& Sancisi, R. 1985, ApJ, 295, 305

Zhang, Y.-Y., Finoguenov, A., Böhringer, H., et al. 2007, A\&A, 467, 437 